\title{
Real-time Out-of-distribution Detection in Learning-Enabled Cyber-Physical Systems
}

\author{
Feiyang Cai \\ Vanderbilt University \\ Nashville, TN \\ feiyang.cai@vanderbilt.edu
}

\author{
Xenofon Koutsoukos \\ Vanderbilt University \\ Nashville, TN \\ xenofon.koutsoukos@vanderbilt.edu
}

\begin{abstract}
Cyber-physical systems (CPS) greatly benefit by using machine learning components that can handle the uncertainty and variability of the real-world. Typical components such as deep neural networks, however, introduce new types of hazards that may impact system safety. The system behavior depends on data that are available only during runtime and may be different than the data used for training. Out-of-distribution data may lead to a large error and compromise safety. The paper considers the problem of efficiently detecting out-of-distribution data in CPS control systems. Detection must be robust and limit the number of false alarms while being computational efficient for real-time monitoring. The proposed approach leverages inductive conformal prediction and anomaly detection for developing a method that has a well-calibrated false alarm rate. We use variational autoencoders and deep support vector data description to learn models that can be used efficiently compute the nonconformity of new inputs relative to the training set and enable realtime detection of out-of-distribution high-dimensional inputs. We demonstrate the method using an advanced emergency braking system and a self-driving end-to-end controller implemented in an open source simulator for self-driving cars. The simulation results show very small number of false positives and detection delay while the execution time is comparable to the execution time of the original machine learning components.
\end{abstract}

Index Terms - anomaly detection, inductive conformal prediction, out-of-distribution, self-driving vehicles.

\section{INTRODUCTION}

Learning-enabled components (LECs) such as neural networks are used in many classes of cyber-physical systems (CPS). Semi-autonomous and autonomous vehicles, in particular, are CPS examples where LECs can play a significant role for perception, planning, and control if they are complemented with methods for analyzing and ensuring safety [1], [2]. However, there are several characteristics of LECs that can complicate safety analysis. LECs encode knowledge in a form that is not transparent. Deep neural networks (DNNs), for example, capture features in a multitude of activation functions that cannot be inspected to ensure that the LEC

The material presented in this paper is based upon work supported by the National Science Foundation (NSF) under grant numbers CNS 1739328, the Defense Advanced Research Projects Agency (DARPA) through contract number FA8750-18-C-0089, and the Air Force Office of Scientific Research (AFOSR) DDDAS through contract number FA9550-18-1-0126. The views and conclusions contained herein are those of the authors and should not be interpreted as necessarily representing the official policies or endorsements, either expressed or implied, of AFOSR, DARPA, or NSF. operates as intended. High levels of autonomy require highcapacity models that further obscure the system operation. Even if an LEC is trained and tested extensively, it is typically characterized by a nonzero error rate. More importantly, the error rate estimated at design-time may be different than the true error because of out-of-distribution data.

Since training data sets are necessarily incomplete, safety assessment at design-time is also incomplete. Design-time verification and analysis methods must be combined with runtime monitoring techniques that can be used for safety assurance. In real-world CPS, the uncertainty and variability of the environment may result in data that are not similar to the data used for training. Although models such as DNNs generalize well if the training and testing data are sampled from the same distribution, out-of-distribution data may lead to large errors. Further, typical DNNs do not have the capability to appropriately estimate if an input is in- or out-of-distribution.

An LEC is trained and tested using data available at designtime but must be deployed in a real system and operate under possibly different conditions. Testing ensures that the error is satisfactory for a large number of examples, however, during the system operation the LEC may still encounter outof-distribution inputs. The proposed approach quantifies how different are the new test data from the training data and raises an alarm to indicate that the LEC may give a prediction with large error. Out-of-distribution detection for CPS must be robust and limit the number of false alarms while being computational efficient for real-time monitoring. Although the paper focuses on DNNs, the approach can be used for other LECs that are designed in a similar fashion.

Detection of out-of-distribution examples in neural networks has received considerable attention especially in the context of classification tasks in computer vision [3]-[5]. Such detection techniques do not take into consideration the dynamical behavior of CPS, can exhibit large number of false alarms, and cannot be applied to CPS in a straightforward manner. Similar techniques based on single input examples are used in mobile robotics [6], [7] where the need for methods to improve robustness is identified as an important research direction.

The proposed approach is based on conformal prediction (CP) [8], [9] and conformal anomaly detection (CAD) [10]. The main idea of these methods is to test if a new input example conforms to the training data set by utilizing a nonconfor- 
mity measure which assigns a numerical score indicating how different the input example is from the training data set. The next step is to define a $p$-value as the fraction of observations that have nonconformity scores greater than or equal to the nonconformity scores of the training examples which is then used for estimating the confidence of the prediction for the test input. In order to use the approach online, Inductive Conformal Anomaly Detection (ICAD) is introduced in [11] where the original training set is split into the proper training set and the calibration set and the $p$-values are computed relative to calibration examples. If a $p$-value is smaller than a predefined anomaly threshold $\epsilon$, the test example can be classified as an anomaly. An important property of the approach is that the rate of detected conformal anomalies is well calibrated, that is with very high probability it is less or approximately equal to a predefined threshold $\epsilon \in(0,1)[11]$. The approach is used for sequential anomaly detection of time trajectories in [10] and for change-point detection in [5], [12]. Existing methods rely on nonconformity measures computed using $k$-Nearest Neighbors and Kernel Density Estimation and cannot scale to LECs with high-dimensional inputs used in CPS.

The main contribution of the paper is real-time detection of out-of-distribution inputs. Our approach leverages inductive conformal prediction and anomaly detection. In order to handle high-dimensional inputs in real-time, we propose to compute the nonconformity scores using learned models based on variational autoencoders (VAEs) [13] and deep support vector data description (SVDD) [14]. VAEs is a generative model which allows sampling multiple examples similar to the input and computing multiple $p$-values that increase the robustness of detection. SVDD is a model trained to perform anomaly detection. In our method, it is combined with a test based on a sliding window that improves the robustness of the detection. By using ICAD, for any valid nonconformity measure, the approach ensures that the rate of detected conformal anomalies is well calibrated. Further, the VAE and SVDD-based methods allow the efficient computation of the nonconformity score and the real-time detection of out-of-distribution high-dimensional inputs. It should be noted that the VAE and SVDD neural networks may exhibit an error different for out-of-distribution inputs that is different than the testing error for in-distribution inputs. However, the robustness of the detection is improved considerably by taking into account multiple input examples and comparing with the calibration nonconformity scores.

Another contribution of the paper is the empirical evaluation using (1) an advanced emergency braking system (AEBS) and (2) a self-driving end-to-end controller (SDEC) implemented in CARLA [15], an open source simulator for self-driving cars. The AEBS uses a perception LEC to detect the nearest front obstacle on the road and estimate the distance from the host vehicle based on camera images. The distance together with the velocity of the host car are used as inputs to a reinforcement learning controller whose objective is to comfortably stop the vehicle. Out-of-distribution inputs are generated by varying a precipitation parameter provided by CARLA which introduces visual effects that may cause large error in the distance estimation resulting to a collision. The simulation results demonstrate a very small number of false positives and a detection delay less than $1 \mathrm{~s}$. For the SDEC which comes with CARLA [15], the empirical evaluation shows that the proposed method can be used to detect a class of physically realizable attacks in end-to-end autonomous driving presented in [16]. The attacks are realized by painted lines on the road to cause the self-driving car to follow a target path. For both examples, the execution time of the detection method is comparable to the execution time of the original LECs which demonstrates that the method can be used in real-time.

\section{System Model And Problem Formulation}

CPS use extensively LECs to perform various tasks in order to increase the level of autonomy. A typical simplified CPS architecture with LECs (e.g., DNNs) for perception and control is shown in Fig. 1. A perception component observes and interprets the environment and provides information to a controller which, possibly using additional sensors (feedback from the plant), applies an action to the plant in order to achieve some task. In response to this action, the state of the physical plant changes and the environment must be observed and interpreted again in order to continue the system operation. An end-to-end control architecture from perception to actuation can also be used.

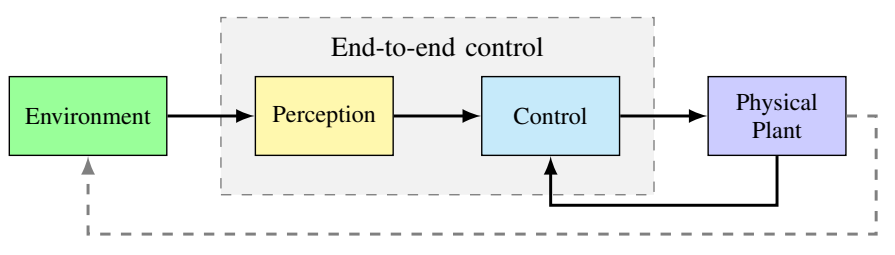

Fig. 1. Simplified CPS control architecture.

An LEC is designed using learning methods such as supervised and reinforcement learning. We assume that the LECs are successfully trained, and further, evaluation of training and testing errors is satisfactory. However, the training and testing data sets at design-time are necessarily incomplete and may under-represent safety critical cases. Out-of-distribution inputs, in particular, that have not been used for training or testing may lead to large errors and compromise safety.

The paper considers the problem of efficiently detecting out-of-distribution inputs in real-time. The objective is to detect such input examples in order enable decision making by switching to a different control architecture or human supervision. During the system operation, the inputs arrive one by one. After receiving each input, the objective is to compute a valid measure of the degree to which the assumption the input example is generated from the same probability distribution as the training data set is falsified.

Evaluation of an online detection must be based on metrics that quantify sensitivity and robustness. Further, out-ofdistribution detection must be performed in real-time which is 
challenging because inputs to perception and end-to-end control LECs are high-dimensional measurements from sensors such as cameras, LIDAR, and RADAR. The time and memory requirements must be similar to the requirements of the LECs used in the CPS architecture.

\section{BACKGROUND}

\section{A. Conformal Prediction and Anomaly Detection}

The proposed approach is based on conformal prediction (CP) [8], [9] and conformal anomaly detection (CAD) [10]. Given a training set $Z=\left\{z_{1}, \ldots, z_{l}\right\}$, the method aims to test if a new test example $z_{l+1}$ conforms to the training data set. Essential in the approach is the definition of a nonconformity measure which is defined by a function $A$ that assigns a numerical score indicating how different the test example is from the training data set. A large nonconformity score corresponds to a strange example relative to the training set. There are many possible functions that can be used [9]. A simple example is the average distance from $z_{l+1}$ to the $k$-nearest neighbors in $Z$ ( $k$-nearest neighbors nonconformity measure). The next step is to define the $p$-value $p_{l+1}$ for the example $z_{l+1}$ based on the nonconformity measure. First, the nonconformity score $\alpha_{i}$ is computed for each example $z_{i}$ relative to the rest $\left\{z_{1}, \ldots, z_{l+1}\right\} \backslash z_{i}$ using $A$, and then, the $p$-value is defined as the fraction of observations that have nonconformity scores greater than or equal to the nonconformity $\alpha_{l+1}$

$$
p_{l+1}=\frac{|\{i=1, \ldots, l\}| \alpha_{i} \geq \alpha_{l+1} \mid}{l} .
$$

The approach is inefficient since all computations have to be repeated for every test example. In order to adapt the approach for online detection, Inductive Conformal Anomaly Detection (ICAD) is introduced in [11] based on Inductive Conformal Prediction (ICP) [9]. The original training set $\left(z_{1}, \ldots, z_{l}\right)$ is split into two sets: the proper training set $\left(z_{1}, \ldots, z_{m}\right)$ and the calibration set $\left(z_{m+1}, \ldots, z_{l}\right)$. For each example in the calibration set, the nonconformity score relative to the proper training set is precomputed by

$$
\alpha_{i}=A\left(\left\{z_{1}, \ldots, z_{m}\right\}, z_{i}\right), i=m+1, \ldots, l .
$$

Then, given a test example $z_{l+1}$, the nonconformity score $\alpha_{l+1}$ is also computed relative to the proper training set and the $p$ value is given by

$$
p_{l+1}=\frac{|\{i=m+1, \ldots, l\}| \alpha_{i} \geq \alpha_{l+1} \mid}{l-m} .
$$

If the $p$-value is smaller than a predefined anomaly threshold $\epsilon \in(0,1)$, the test example is classified as a conformal anomaly. In this case, the test example can be a rare, previously unseen example from the same probability distribution as the training data set, an out-of-distribution example, or the training examples are not independent and identically distributed (IID). The approach can be viewed as a statistical hypothesis test, where the null hypothesis that the new example $z_{l+1}$ and the training set $\left(z_{1}, \ldots, z_{l}\right)$ are IID is tested at significance level $\epsilon$. If $z_{l+1}$ and $\left(z_{1}, \ldots, z_{l}\right)$ are in fact IID, then for any choice of nonconformity measure, $\epsilon$ is an upper bound of the probability that $z_{l+1}$ is classified as a conformal anomaly. An important property of the approach is that the rate of detected conformal anomalies is well calibrated, that is with very high probability it is less or approximately equal to $\epsilon$ [11].

Conformal prediction and anomaly detection can be applied under the exchangeability assumption which is weaker than the IID assumption. Consider a sequence $\left\{z_{1}, z_{2}, \ldots, z_{N}\right\}$ generated from a probability distribution that is exchangeable, then for any permutation $\pi$ of the set $\{1, \ldots, N\}$, the distribution of $\left\{z_{\pi(1)}, z_{\pi(2)}, \ldots, z_{\pi(N)}\right\}$ is the same as the distribution of the original sequence [9]. Consider $\left\{z_{1}, z_{2}, \ldots, z_{N}\right\}$ generated from the same exchangeable probability distribution as the proper training set. It is shown in [8] (Theorem 8.2), that the $p$-values $p_{1}, p_{2}, \ldots, p_{N}$ are independent and uniformly distributed in $[0,1]$. The sequence of $p$-values can be used to test online if the observations $\left\{z_{1}, z_{2}, \ldots, z_{N}\right\}$ are generated from the same probability distribution as the proper training set since the algorithm will generate small $p$ values for unusual examples.

Testing the hypothesis that $p$-values are independent and uniformly distributed can be performed using martingales that are constructed using the $p$-values [17]. Given $p_{1}, p_{2}, \ldots, p_{N}$, [17] proposes the power martingale defined for some $\epsilon$ as

$$
M_{N}^{\epsilon}=\prod_{i=1}^{N} \epsilon p_{i}^{\epsilon-1}
$$

and the simple mixture martingale defined as

$$
M_{N}=\int_{0}^{1} M_{N}^{\epsilon} d \epsilon .
$$

Such a martingale will grow only if there are many small $p$ values in the sequence. If the generated $p$-values concentrate in any other part of the unit interval, the martingale is not expected to grow. Details and other martingales that follow the same idea can be found in [17].

Application of the approach relies on the nonconformal measure which must be computed efficiently and score correctly the strangeness of high-dimensional examples. Typical nonconformity measures such as the $k$-Nearest Neighbor $(k$ NN) nonconformity measure [11] and the Kernel Density Estimation (KDE) nonconformity measure [5] cannot scale efficiently to high-dimensional inputs because they require either storing the training data set or estimating the density in a high-dimensional space. One of the main contributions of the paper is computing the nonconformity measures efficiently using learned models based on variational autoencoders [13] and deep support vector data description [14].

\section{B. Variational Autoencoders}

Variational Autoencoder (VAE) is a generative model which learns parameters of a probability distribution to represent the data [13]. A VAE consists of an encoder, a decoder, and a loss function. The objective is to model the relationship between 
the observation $z$ and the low-dimensional latent variable $x$ using the loss function

$$
\mathcal{L}(\theta, \phi ; z)=\mathbb{E}_{x \sim q_{\phi}(x \mid z)}\left[\log p_{\theta}(z \mid x)\right]-D_{\mathrm{KL}}\left[q_{\phi}(x \mid z) \| p(x)\right],
$$

where $\theta$ and $\phi$ are neural network parameters. The first term is the model fit and the second is the $\mathrm{KL}$ divergence between the approximate posterior and the prior of $x$. A popular choice for the prior is Gaussian distribution. VAE-based methods can utilize the reconstruction error or reconstruction accuracy for anomaly detection [18]. In our approach, given an input $z$ the generative model (decoder) is used to sample IID examples from $q_{\phi}(x \mid z)$ and the reconstruction accuracy is used as a nonconformity measure.

\section{Deep Support Vector Data Description}

VAEs and other autoencoder architectures are trained to perform a task other than anomaly detection assuming that the reconstruction accuracy will be better for in-distribution examples. Deep Support Vector Description (SVDD) is an architecture trained to perform anomaly detection [14]. The idea is to train a DNN to map the input data into a hypersphere of minimum volume characterized by center $c$ and radius $R$. The input space $\mathcal{Z}$ is transformed to a compressed output space $\mathcal{X}$ while minimizing the volume of the hypersphere that encloses most of the input representations. Given a training data set $\left\{z_{1}, \ldots, z_{n}\right\}$, the one-class deep SVDD [14] is based on the loss

$$
\min _{\mathcal{W}} \frac{1}{n} \sum_{i=1}^{n}\left\|\phi\left(z_{i} ; \mathcal{W}\right)-\boldsymbol{c}\right\|^{2}+\frac{\lambda}{2} \sum_{\ell=1}^{L}\left\|\boldsymbol{W}^{\ell}\right\|_{F}^{2},
$$

where $\phi(\cdot ; \mathcal{W}): \mathcal{Z} \rightarrow \mathcal{X}$ denotes the neural network with weights $\mathcal{W}, c \in \mathcal{X}$ is the center of the hypersphere, and the last term is a weight regularizer with hyperparameter $\lambda>0$, where $\|\cdot\|_{F}$ is the Frobenius norm. One-class deep SVDD learns to map the data as close to center $c$ as possible by penalizing the distance from representations to the center. The deep SVDD neural network must not have bias terms or bounded activation functions and the center $c$ can be selected as the mean of the representations from the initial inference on some training data to avoid trivial solutions that map the input space to a single point [14]. Given a new test example $z$, the distance of the representation $\phi\left(z ; \mathcal{W}^{*}\right)$ to the center $c$ of the hypersphere reflects how different the test example is from the training data set and can be used as a nonconformity measure.

\section{OUT-OF-DISTRIBUTION DETECTION}

\section{A. Detection Algorithm}

The algorithm is based on the ICP and ICAD, and therefore, the training data set is split into a proper training set $\left(z_{1}, \ldots, z_{m}\right)$ and a calibration set $\left(z_{m+1}, \ldots, z_{l}\right)$. Practically, the data set used for training the LEC can be used as the proper training set, and after the LEC training phase, additional data can be collected to form the calibration set. For each example in the calibration set, a function $A$ is used to compute the nonconformity measure. The nonconformity scores of the calibration examples are sorted in order to be used at runtime. Algorithm 1 shows the steps that are performed offline.

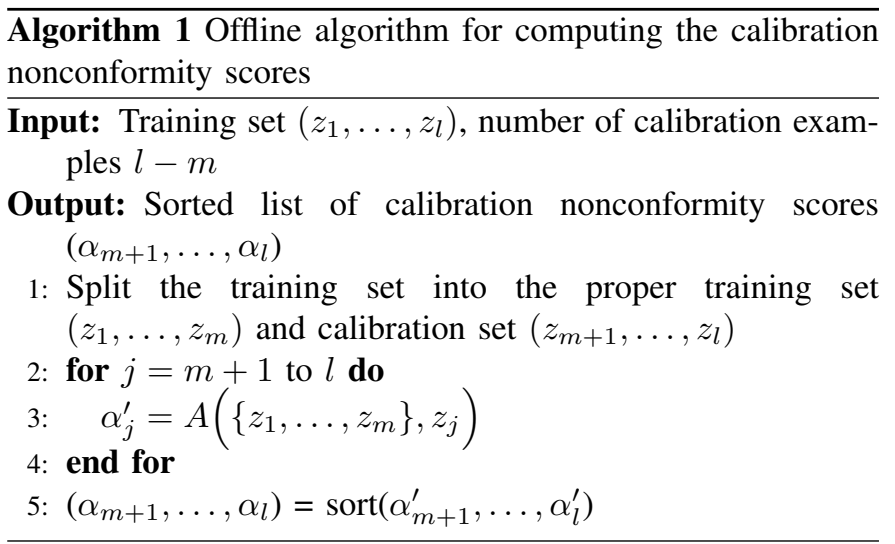

Let us consider an LEC defining a mapping from input $z$ to output $y$. The set of input examples used for training is denoted by $\left(z_{1}, \ldots, z_{l}\right)$. During the system operation, a sequence of inputs denoted by $\left(z_{1}^{\prime}, \ldots, z_{N}^{\prime}\right)$ is processed one-by-one. The task of the algorithm is to quantify how different the input sequence is from the training data set. If the difference is large, the algorithm raises an alarm indicating that the LEC may generate an output $y$ with large error compared to the testing error obtained at design-time.

At runtime, given a new input $z_{k}^{\prime}$, the noncomformity score $\alpha_{k}^{\prime}$ can be computed using the nonconformity function $A$ relative to the proper training set

$$
\alpha_{k}^{\prime}=A\left(\left\{z_{1}, \ldots, z_{m}\right\}, z_{k}^{\prime}\right) .
$$

The computation requires evaluating the strangeness of $z_{k}^{\prime}$ relative to $\left\{z_{1}, \ldots, z_{m}\right\}$. The choice of the nonconformity function $A$ must ensure computing informative nonconformity scores in real-time. Using, for example, $k$-NN requires storing the training data set which is infeasible for real-world CPS. Instead, we propose to learn an appropriate neural network architecture which is trained offline using the proper training set and encodes the required information in its parameters. This neural network monitors the inputs to the perception or end-to-end control LEC and is used to compute in real-time the nonconformity measure.

Given an input $z_{k}^{\prime}$, the $p$-value $p_{k}$ is computed as the fraction of calibration examples that have nonconformity scores greater than or equal to $\alpha_{k}^{\prime}$ using Eq. (1). It should be noted that the computation of the $p$-value can be performed efficiently online since it requires storing only the calibration nonconformity scores. If $p_{k}<\epsilon$ the example $z_{k}^{\prime}$ is classified as an anomaly. Using a single $p$-value for detecting out-ofdistribution examples can lead to an oversensitive detector with a large number of false alarms that inhibit the operation of the CPS. Our objective is to compute a sequence of $p$-values and use the martingale-based method presented in Section III to test if the $p$-values are independent and uniformly distributed indicating that the input is generated from the same probability distribution as the proper training data set or there are many 
small $p$-values indicating an out-of-distribution input. In the latter case, the martingale will grow and can be used as an input to a detector that raises alarms.

In the following, we describe how VAE and SVDD are used to compute the nonconformity measure and detect out-of distribution examples. Although the idea is similar, the two architectures lead to different algorithms for computing the sequence of $p$-values and realizing the detector.

\section{B. VAE-based Out-of-distribution Detection}

1) Nonconformity measure and p-values: Given an input example $z_{t}$ at time $t$, the encoder portion of a VAE is used to approximate the posterior distribution of the latent space and sample multiple points $x_{k}$ from the posterior that are used as input to the decoder portion in order to and generate new examples $z_{1}^{\prime}, \ldots, z_{N}^{\prime}$. Typically, the posterior of the latent space is approximated by a Gaussian distribution. Sampling from the posterior generates encodings $x_{k}$ so that the decoder is exposed to a range of variations of the input example and outputs $z_{1}^{\prime}, \ldots, z_{N}^{\prime}$ which satisfy the exchangeability assumption.

An in-distribution input $z_{t}$ should be reconstructed with a relatively small reconstruction error. Conversely, an outof-distribution input will likely have a larger error. The reconstruction error is a good evaluation of the strangeness of the input relative to the training set and it is used as the nonconformity measure. We use the squared error between the input example $z_{t}$ and each generated output example $z_{k}^{\prime}$ as the nonconformity measure defined as

$$
\alpha_{k}^{\prime}=A_{\mathrm{VAE}}\left(z_{t}, z_{k}^{\prime}\right)=\left\|z_{t}-z_{k}^{\prime}\right\|^{2} .
$$

The $p$-value $p_{k}$ for the input $z_{k}^{\prime}$ is computed as the fraction of calibration examples that have nonconformity scores greater than or equal to $\alpha_{k}^{\prime}$ using Eq. (1). Since the examples $z_{1}^{\prime}, \ldots, z_{N}^{\prime}$ satisfy the exchangeability assumption, the $p_{k}$ values are independent and uniformly distributed in $[0,1]$ (see discussion in Section III) and the martingale method can be used to test if $z_{1}^{\prime}, \ldots, z_{N}^{\prime}$, and therefore $z_{t}$, are generated from the probability distribution of the training data.

2) Martingale test: At runtime, for every new input example $z_{t}$ received by the perception or end-to-end control LEC at time $t$ we compute the martingale

$$
M_{t}=\int_{0}^{1} M_{t}^{\epsilon} d \epsilon=\int_{0}^{1} \prod_{k=1}^{N} \epsilon p_{k}^{\epsilon-1} d \epsilon .
$$

$M_{t}$ will have a large value if there are many small $p$-values in the sequence $p_{k}$ which will indicate an out-of-distribution input.

3) Stateful detector: In order to robustly detect when $M_{t}$ becomes consistently large, we use the Cumulative sum (CUSUM) procedure [19]. CUSUM is a nonparametric stateful test and can be used to generate alarms for out-of-distribution inputs by keeping track of the historical information of the martingale values.

The detector is defined as $S_{1}=0$ and $S_{t}=\max \left(0, S_{t-1}+\right.$ $M_{t-1}-\delta$ ), where $\delta$ prevents $S_{t}$ from increasing consistently when the inputs are in the same distribution as the training data. An alarm is raised whenever $S_{t}$ is greater than a threshold $S_{t}>\tau$ which can be optimized using empirical data [19]. Typically, after an alarm the test is reset with $S_{t+1}=0$.

Algorithm 2 describes the VAE-based real-time out-ofdistribution detection. The nonconformity measure can be computed very efficiently by executing the learned VAE neural network and generating $N$ new examples. The complexity is comparable to the complexity of the perception or end-to-end LEC that is executed in real-time.

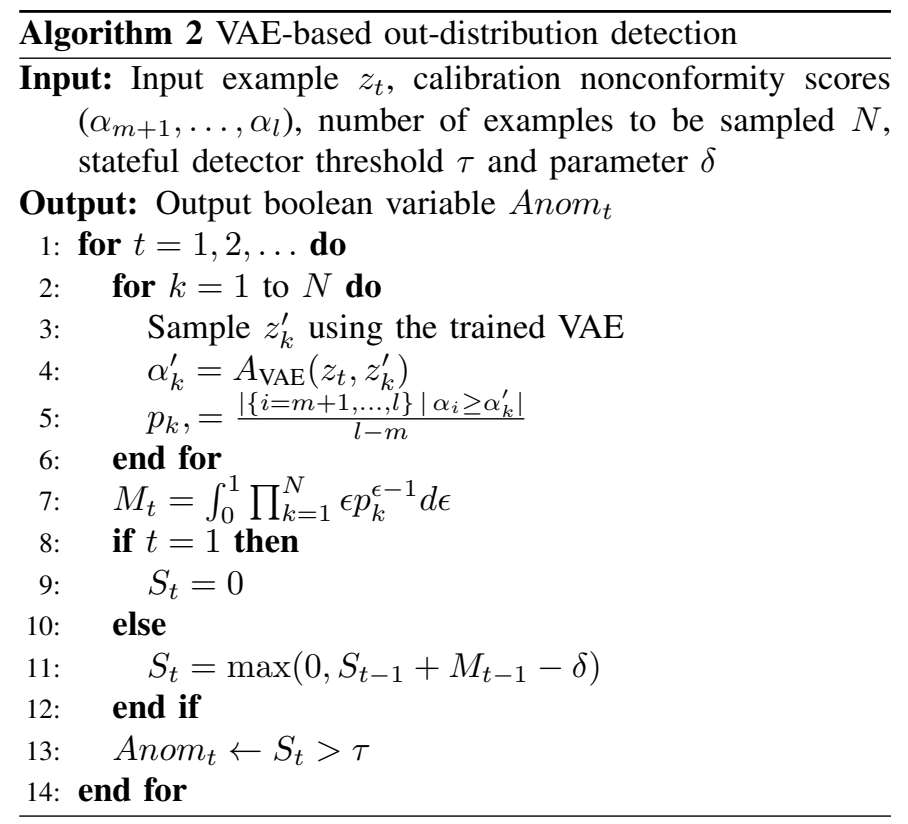

\section{SVDD-based out-of-distribution Detection}

1) Nonconformity measure and p-values: The SVDD-based method also uses a learned model to calculate the nonconformity score. The proper training set is used to train the deep SVDD model. The center of the hypersphere $c$ is fixed as the mean of the representations from the initial pass on the proper training data. After training, the neural network function $\phi\left(z_{t}, \mathcal{W}^{*}\right)$ maps an input example $z_{t}$ to a representation close to the center $c$. In-distribution inputs are likely concentrated in a relatively small area in the output space while the out-of-distribution inputs will be faraway from the center. The distance of the representation to the center $c$ of the hypersphere can be used to evaluate the strangeness of the test example relative to the proper training set and is defined as the nonconformity measure

$$
\alpha_{t}^{\prime}=A_{\mathrm{SVDD}}\left(z_{t}\right)=\left\|\phi\left(z_{t} ; \mathcal{W}^{*}\right)-\boldsymbol{c}\right\|^{2} .
$$

The $p$-value is computed as the fraction of calibration examples that have nonconformity scores greater than or equal to $\alpha_{t}^{\prime}$ (Eq. 11). However, in contrast to the VAE, SVDD is not a generative model and cannot be used to generate multiple IID examples similar to $z_{t}$. 
2) Martingale test: In order to improve the robustness of out-of-distribution detection, it is desirable to use a sequence of inputs. In CPS, the inputs arrive at the perception or end-to-end LEC one-by-one and they are time-correlated, and therefore not independent. For a sequence of inputs $z_{t}: t=1,2, \ldots$, the martingale in Eq. (2) will increase continuously even for in-distribution examples. In order to adapt the test, we use a sliding window $[t-N+1, t]$, and given an input sequence $\left(z_{t-N+1}, \ldots, z_{t}\right)$, we compute the sequence of $p$-values $\left(p_{t-N+1}, \ldots, p_{t}\right)$. Although the $p$-values are not guaranteed to be independent and uniformly distributed, outof-distribution inputs will still result in small $p$-values and the martingale test is used to identify sequences with many small values. In this case, the martingale is given by

$$
M_{t}=\int_{0}^{1} M_{t}^{\epsilon} d \epsilon=\int_{0}^{1} \prod_{i=t-N+1}^{t} \epsilon p_{i}^{\epsilon-1} d \epsilon .
$$

In order to apply this method to CPS, the rate that we receive observations from the environment must be much faster than the dynamic evolution of the system and the main factor that differentiates consecutive observations are random disturbances and noise. For a short window, it can be assumed that the input sequence $\left(z_{t-N+1}, \ldots, z_{t}\right)$ satisfies the exchangeability assumption and the martingale test can be used to detect multiple small $p$-values in a short time interval. It should be noted that the martingale $M_{t}$ does not depend on the order of the input examples $\left(z_{t-N+1}, \ldots, z_{t}\right)$. Also, $M_{t}$ must be initialized for the first steps using, for example, random independent and uniformly distributed $p$-values.

3) Stateless detector: Since we already use a sliding window to compute $M_{t}$, we employ a stateless detector based on the value $M_{t}$ and a predefined thershold $\tau$ expressed as $M_{t}>\tau$

Algorithm 3 describes the SVDD-based real-time out-ofdistribution detection. Compared with the VAE, the SVDD based method is more efficient since it does not require generating multiple examples at each step. The martingale $M_{t}$ can be computed recursively by incorporating the $p$-value for the new input and omitting the last one in the sliding window.

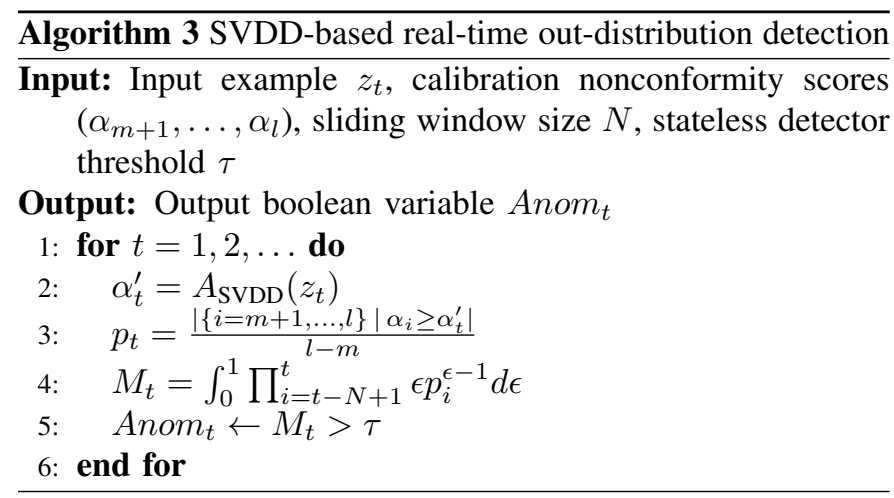

\section{Evaluation}

We evaluate the proposed approach using (1) an advanced emergency braking system (AEBS) and (2) a self-driving end-to-end controller (SDEC). The AEBS and SDEC are implemented using CARLA [15]. We use CARLA 0.9.5 on a 16-core i7 desktop with $32 \mathrm{~GB}$ RAM memory and a single RTX 2080 GPU with 8 GB video memory.

\section{A. Advanced Emergency Braking System}

1) Experimental Setup: The architecture of the AEBS is shown in Fig. 2. A perception LEC is used to detect the nearest front obstacle on the road and estimate the distance. The distance together with the velocity of the host car are used as inputs to a reinforcement learning controller whose objective is to generate the appropriate braking force in order to safely and comfortably stop the vehicle.

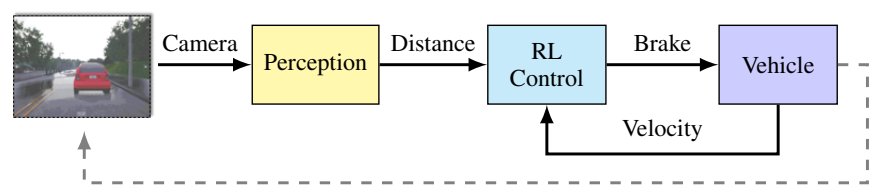

Fig. 2. Advanced emergency braking system architecture.

The desirable behavior is illustrated in Fig. 3. The AEBS detects a stopped lead car and applies the brake to decelerate and avoid the potential collision. The initial velocity of the host vehicle is $v_{0}$ and the initial distance between the host car and the obstacle is $d_{0}$. The goal of the controller is to stop the car between $L_{\min }$ and $L_{\max }$. The sampling period used in the simulation is $\Delta t=1 / 20 \mathrm{~s}$. In order to simulate realistic scenarios, we introduce uncertainty into the system. The initial velocity $v_{0}$ is uniformly sampled between $90 \mathrm{~km} / \mathrm{h}$ and $100 \mathrm{~km} / \mathrm{h}$, and the initial distance $d_{0}$ is approximately $100 \mathrm{~m}$. CARLA allows controlling the precipitation in the simulation using a parameter which takes values in $[0,100]$. For training the perception LEC, and also the VAE and SVDD used for out-of-distribution detection, the precipitation parameter is randomly sampled from the interval $[0,20]$. The uncertainty introduced affects the error of the perception LEC. It should be noted that this is just a visual effect and it does not affect the physical behavior of the car.

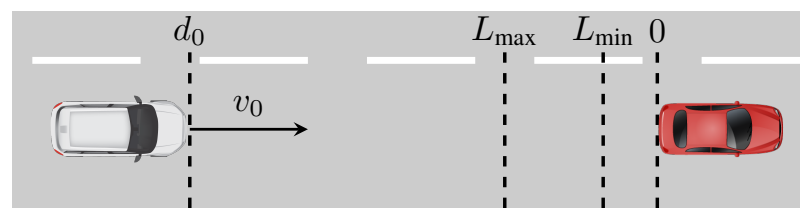

Fig. 3. Illustration of advanced emergency braking system.

The perception LEC is implemented using a convolutional neural network (CNN) which is trained using supervised learning with a training data set consisting of 8160 images obtained by varying the simulation parameters described above. The perception LEC has three layers of $24 / 36 / 48 \times(5 \times 5)$ filters with ReLU activations and $2 \times 2$ strides, two layers of $64 / 64 \times(3 \times 3)$ filters with ReLU activations and $1 \times 1$ 
strides, three fully connected layers of 100/50/10 units with ReLU activations and an output layer of size 1 with Sigmoid activation. After 100-epoch training, the mean absolute errors for training and testing are $0.54 \mathrm{~m}$ and $0.56 \mathrm{~m}$ respectively and are used to select $L_{\min }$ and ensure safety. The reinforcement learning controller is trained using the DDPG algorithm [20] with 1000 episodes and reward function which aims to stop the vehicle between $L_{\min }=1 \mathrm{~m}$ and $L_{\max }=3 \mathrm{~m}$. A simulation run is shown in Fig. 4 Initially, the distance between the host and the lead car is $97.56 \mathrm{~m}$, and the velocity of the host car is $96.84 \mathrm{~km} / \mathrm{h}(=26.90 \mathrm{~m} / \mathrm{s})$. After 140 steps or $7.00 \mathrm{~s}$, the host vehicle stops at $1.98 \mathrm{~m}$ from the lead car.

2) VAE and SVDD training: The data set with the 8160 images used for training the perception LEC is used as the proper training data set. In addition, using simulations with the same random parameters, we collect 2040 images for the calibration set. We use a VAE with four layers of $32 / 64 / 128 / 256 \times(5 \times 5)$ filters with exponential linear unit (ELU) activations and $2 \times 2$ max-pooling, one fully connected layer of size 1568 with ELU activation, 1024 latent space, and a symmetric deconvolutional decoder. A simple two-phase learning schedule is employed with initial searching learning rate $\eta=10^{-4}$ for 250 epochs, and subsequently fine-tuning $\eta=10^{-5}$ for 100 epochs.

The Deep SVDD is similar with four convolutional layers of $32 / 64 / 128 / 256 \times(5 \times 5)$ filters with ELU activations and $2 \times 2$ max-pooling, followed by one fully connected layer of 1568 units. As suggested in [14], we first train a deep convolutional autoencoder (DCAE) to initialize the deep SVDD. After $250\left(\eta=10^{-4}\right)+100\left(\eta=10^{-5}\right)$ epochs of DCAE training, we copy the weights to the SVDD and set the hypersphere center $c$ to the mean of the reduced space of the initial forward inference. The one-class deep SVDD objective is used as the loss and the neural network is trained for additional $150\left(\eta=10^{-4}\right)+100\left(\eta=10^{-5}\right)$ epochs.

3) Results: To characterize the performance of the outof-distribution detection, we use multiple simulation episodes that include in- and out-of-distribution examples. Each episode starts with a random initial velocity $v_{0}$ of the host car. The AEBS is activated upon detection of the lead car by the camera as implemented in CARLA. We vary the precipitation parameter $r$ as

$$
r= \begin{cases}r_{0} & \text { for } t<t_{0} \\ r_{0}+\beta\left(t-t_{0}\right) & \text { for } t_{0} \leq t \leq t_{1} \\ r_{0}+\beta\left(t_{1}-t_{0}\right) & \text { for } t>t_{1}\end{cases}
$$

where $r_{0}$ is the initial precipitation uniformly sampled from $[0,10] ; t_{0} \in\{10,11, \ldots, 30\}$ is selected randomly as the time step the precipitation starts to increase; $t_{1} \in\{90,91, \ldots, 110\}$ is selected randomly as the time step the precipitation stops increasing; and $\beta \in[0.1,0.5]$ is a randomly selected slope. In some episodes $r$ is always below 20 (in-distribution) while in other episodes $r$ exceeds 20 and it is assumed that the perception LEC receives out-of-distribution inputs. We simulate 200 episodes and 108 of them are in-distribution while 92 of them contain out-of-distribution inputs.
We illustrate the approach using two episodes and we plot the ground truth and the predicted distance to the lead car, the velocity of the host car, the $p$-value and stateful detector $S$-value computed using the logarithm of $M_{t}$ and $\delta=6$, the $p$-value of the SVDD-based method, and the logarithm of the SVDD-based martingale. Since $M_{t}$ takes very large values, $\log M_{t}$ is used. We use $N=10$ for the number of examples generated by the VAE and the size of sliding window in the SVDD-based approach. Fig. 4 shows simulation results for the in-distribution case. The $p$-values are randomly distributed between 0 to 1 , and the martingale for both approaches is small. The VAE-based method is more sensitive than the SVDD as indicated by the larger value around $5 \mathrm{~s}$. The $p$ values may become small if the camera observations are different than the training data. In this case, there is a speed limit traffic sign that is not accurately reconstructed. After the car passes the traffic sign, the $p$-values increase and the martingale goes back to very small value. The effect in the SVDD method is smaller, since we use a sliding window. Even if the training data set is augmented, there will be always novelties that can lead to variation but a robust detection method can limit the number of false alarms.

An episode with out-of-distribution inputs is shown in Fig. 5. The parameter $r$ exceeds 20 at time step $40(2.0 \mathrm{~s})$. The error of the perception LEC starts increasing and reaches almost $11 \mathrm{~m}$. The controller is misled by the perception LEC, and does not stop the car which collides with the lead car (velocity is greater than 0 when ground truth distance comes to 0). Both VAE-based and SVDD-based martingale grow as the $p$-values become smaller.

We evaluate the approach for the 200 episodes generated by considering different values of $N$. We run each episode and if an alarm is raised, we stop the simulation, and we check if the alarm is false. We compute the detection delay as the number of frames from the time $r$ exceed 20. We select the detector parameters $\tau$ and $\delta$ using a simple search for achieving average detection delay less than 25 frames. Tables I and II] shows the results for the VAE and SVDD-based methods respectively. The number of false alarms is very small and the delay for detection is smaller than 20 frames or $1 \mathrm{~s}$.

TABLE I

VAE-BASED DETECTION.

\begin{tabular}{cccc}
\hline $\begin{array}{c}\text { Parameters } \\
(N, \delta, \tau)\end{array}$ & False positive & False negative & $\begin{array}{c}\text { Average delay } \\
\text { (frames) }\end{array}$ \\
\hline $5,5,42$ & $2 / 108$ & $0 / 92$ & 17.91 \\
\hline $5,5,49$ & $0 / 108$ & $0 / 92$ & 19.84 \\
\hline $10,6,156$ & $0 / 108$ & $0 / 92$ & 18.65 \\
\hline $10,10,106$ & $0 / 108$ & $0 / 92$ & 19.30 \\
\hline $20,16,250$ & $2 / 108$ & $0 / 92$ & 17.63 \\
\hline $20,18,240$ & $0 / 108$ & $0 / 92$ & 18.46 \\
\hline
\end{tabular}

\section{B. Self-driving End-to-end Control}

1) Experimental Setup: The CARLA simulator comes with a self-driving end-to-end controller trained using imitation learning. The SDEC uses camera images as inputs and 


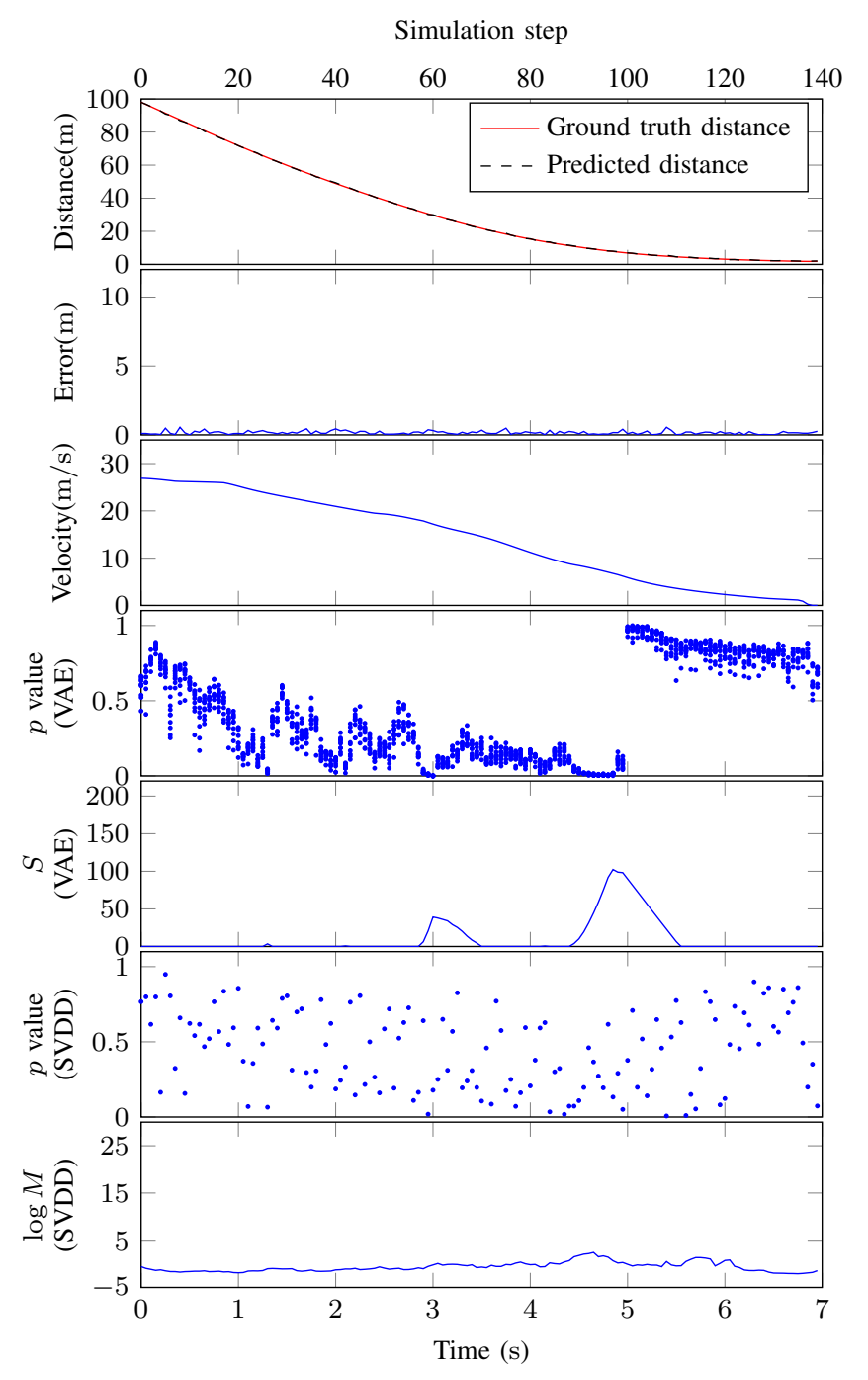

Fig. 4. Episode with in-distribution inputs.

TABLE II

SVDD-BASED DETECTION.

\begin{tabular}{cccc}
\hline $\begin{array}{c}\text { Parameters } \\
(N, \tau)\end{array}$ & False positive & False negative & $\begin{array}{c}\text { Average delay } \\
\text { (frames) }\end{array}$ \\
\hline 5,8 & $2 / 108$ & $0 / 92$ & 15.12 \\
\hline 5,9 & $1 / 108$ & $0 / 92$ & 20.85 \\
\hline 10,12 & $1 / 108$ & $0 / 92$ & 14.38 \\
\hline 10,14 & $0 / 108$ & $0 / 92$ & 17.78 \\
\hline 20,16 & $1 / 108$ & $0 / 92$ & 12.02 \\
\hline 20,17 & $0 / 108$ & $0 / 92$ & 13.29 \\
\hline
\end{tabular}

computes steering, acceleration, and brake actuation signals applied to the car. The SDEC is implemented using a CNN trained by conditional imitation learning with 14 hours of driving data recorded by human drivers [15]. The sampling period used here is $\Delta t=1 / 10 \mathrm{~s}$. For this example, our objective is to evaluate if the method can be used to detect a class of adversarial attacks. An approach for designing physically realizable attacks in end-to-end autonomous driving is presented in [16] and a novel class of hijacking attacks is

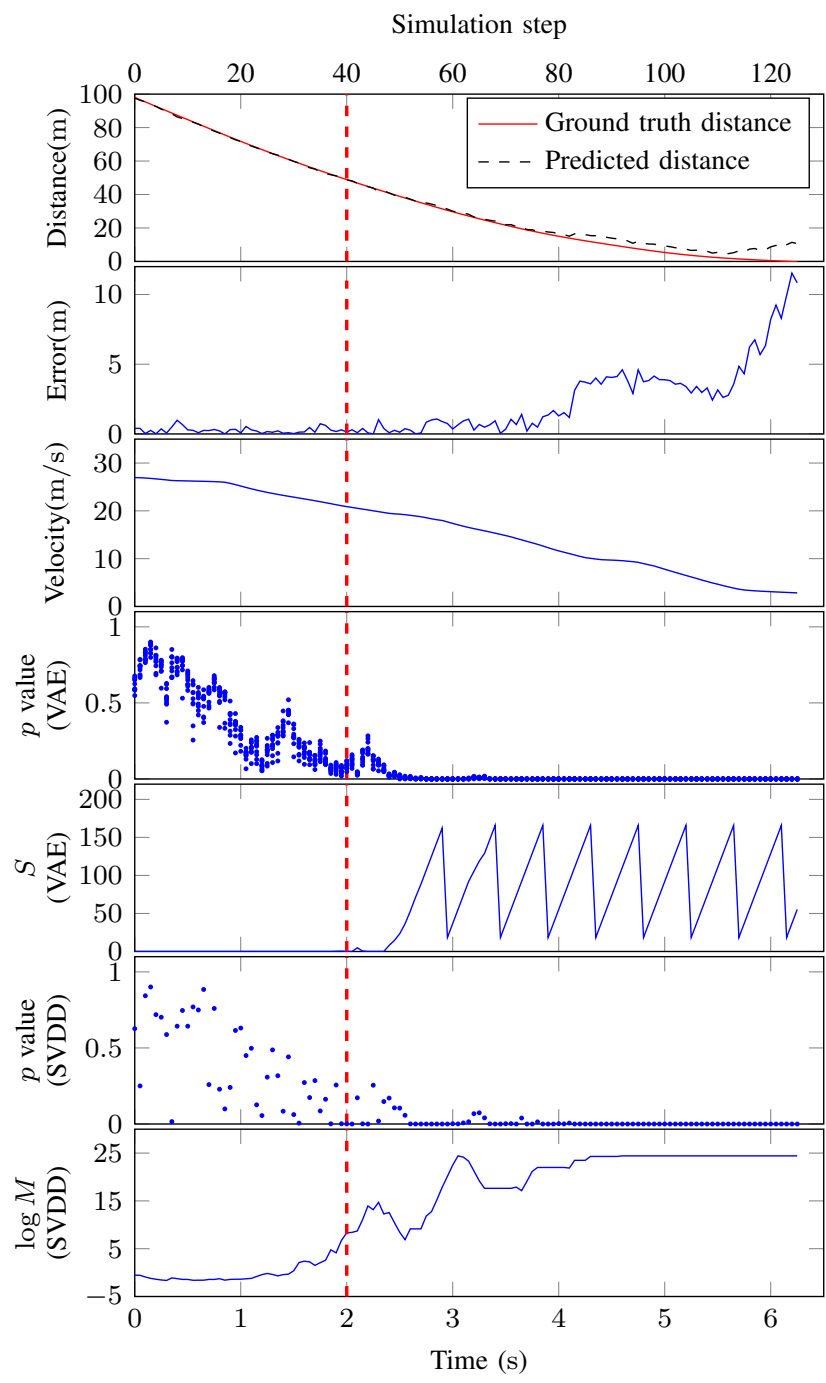

Fig. 5. Episode with out-of-distribution inputs.

introduced where painted lines on the road cause the selfdriving car to follow a target path. Fig. 6b shows an image with the painted pattern on the road.

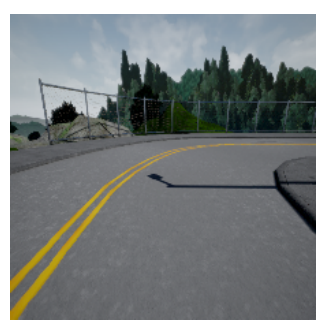

(a) Original image

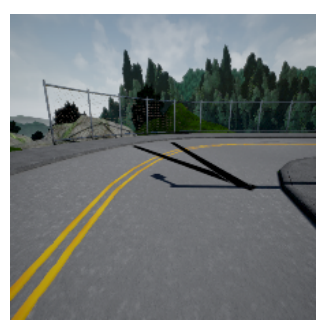

(b) Attacked image
Fig. 6. Comparison of original image and image with attack [16].

In order to train the VAE and SVDD, we collect training data using episodes without attacks. We generate 633 images in two different weather patterns (clear noon and cloudy noon) and three different scenarios (turning right, turning left, and 
going straight). We randomly split the training data into 506 images for the proper training data set and 127 images for the calibration set. We use the same VAE and SVDD architectures and hyperparameters as in the AEBS.

The evaluation focuses on the Right Corner Driving case which is reported as more vulnerable [16]. We run 105 simulation episodes described in [16] with different attacks such as positions and rotations of the two black lines which are chosen to cause traffic infractions. In 69 out of the 105 episodes the attack is successful causing a vehicle crash. Our approach detects the attacks in all 105 episodes. Distinguishing between attacks that cause a crash or not using only the input images is an interesting question for further research. Also, it is important to investigate how to design attacks that hijack both the end-to-end control and the out-of-distribution detection.

We plot the $p$-values and stateful detector $S$-value of the VAE-based method, the $p$-values and the logarithm of the SVDD-based martingale in Fig. 7 ( $N=10$ and $\delta=1)$. In this episode, there are two black lines painted on the road as shown in Fig. 6b and the vehicle is misled leading to a crash. The $p$-values are almost 0 and the martingales grow very large indicating the input images are out-of-distribution.

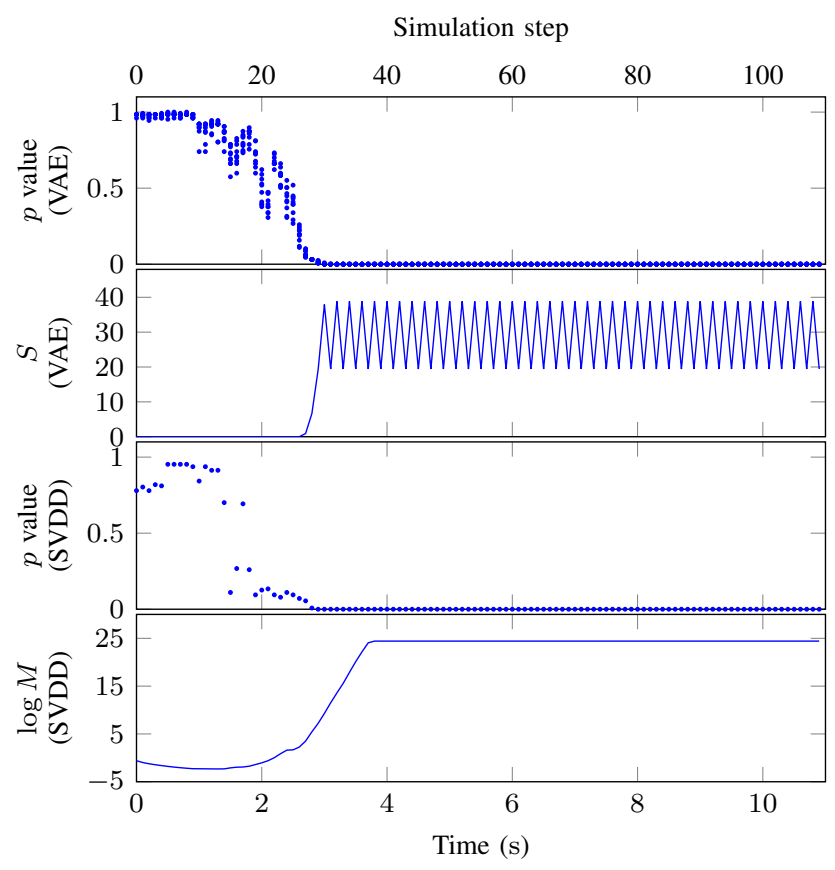

Fig. 7. Episode with attacked inputs.

\section{Computational Efficiency}

The VAE-based and SVDD-based methods can compute the nonconformity scores in real-time without storing training data. Table III reports the minimum (min), first quartile $\left(Q_{1}\right)$, second quartile or median $\left(Q_{2}\right)$, third quartile $\left(Q_{3}\right)$, and maximum (max) of (1) the execution times of the LECs in AEBS and SDEC and (2) the execution times of the VAE-based and SVDD-based detectors for different values of $N$. Since the VAE-based method uses $N$ examples in each time step to compute the nonconformity scores, the execution time is larger than the execution time of the SVDD-based method. The execution time of SVDD-based detection method is independent of the window size $N$ since the martingale can be computed recursively for the sliding window. The execution times are similar to the execution times of the perception and end-to-end control LECs and much smaller than the corresponding sampling time $(50 \mathrm{~ms}$ in AEBS and $100 \mathrm{~ms}$ in SDEC), and thus, the methods can be used for realtime out-of-distribution detection.

TABLE III

EXECUTION TIMES.

\begin{tabular}{ccccccc}
\hline & $N$ & $\begin{array}{c}\min \\
(\mathrm{ms})\end{array}$ & $\begin{array}{c}Q_{1} \\
(\mathrm{~ms})\end{array}$ & $\begin{array}{c}Q_{2} \\
(\mathrm{~ms})\end{array}$ & $\begin{array}{c}Q_{3} \\
(\mathrm{~ms})\end{array}$ & $\begin{array}{c}\max \\
(\mathrm{ms})\end{array}$ \\
\hline AEBS & N/A & 3.48 & 3.85 & 3.91 & 3.96 & 4.20 \\
\hline SDEC & N/A & 2.20 & 2.37 & 2.45 & 2.56 & 3.31 \\
\hline \multirow{3}{*}{ VAE } & 5 & 18.64 & 18.68 & 18.71 & 18.73 & 18.81 \\
& 10 & 37.26 & 37.38 & 37.41 & 37.45 & 38.11 \\
& 20 & 75.12 & 75.19 & 75.22 & 75.68 & 78.83 \\
\hline \multirow{3}{*}{ SVDD } & 5 & 2.18 & 2.19 & 2.19 & 2.2 & 2.26 \\
& 10 & 2.17 & 2.19 & 2.19 & 2.2 & 2.29 \\
& 20 & 2.17 & 2.22 & 2.23 & 2.23 & 2.37 \\
\hline
\end{tabular}

\section{RELATED WORK}

Verification and assurance of CPS with machine learning components is considered in [21] in a broader context of verified artificial intelligence. The challenges discussed in [21] include the integration of design-time and runtime methods to address the undecidability of verification in complex systems and environment modeling. Out-of-distribution detection can be used with recovery and reconfiguration techniques to complement design-time verification. Focusing on design-time techniques, an approach to identify regions of the input space that lack training data and potentially larger errors is presented in [22]. The approach could be adapted to predict at runtime if new inputs are from regions covered during training or not. Compositional falsification of CPS with machine learning components is introduced in [2] and demonstrated with a simulated AEBS. The approach is applied at design-time for identifying executions that falsify temporal logic specifications and also identifies regions of uncertainty where additional analysis and runtime monitoring is required. A related approach for simulation-based adversarial test generation for autonomous vehicles with machine learning components is presented in [1]. The technique is also used at design-time to increase the reliability of autonomous CPS and can provide additional training data for out-of-distribution detection.

Detection of out-of-distribution examples in neural networks has received considerable attention especially in the context of classification tasks in computer vision [3]. Correctly classified examples tend to have greater maximum softmax probabilities than erroneously classified and out-of-distribution examples. An approach for improving detection by training anomaly detectors is proposed in [4]. An approach for reducing the number of false alarms of out-of-distribution image detection by adding small perturbations to the input is presented in [23]. 
The idea is similar to randomly sampling from the latent space of the VAE that can also improve the reliability of the detection. Such detection techniques do not take into consideration the dynamical behavior of CPS and can exhibit large number of false alarms.

An approach which aims to detect novelties based on the reconstruction error of an autoencoder for a single input is presented in [6]. The approach is used for safe visual and LIDAR-based navigation of mobile robots. A similar approach using VAEs is proposed in [7] to estimate the uncertainty for a collision prediction task for a robot car. As discussed in [6], [7], out-of-distribution detection and, in general, uncertainty estimation is an important research direction for providing a more robust detection. In our approach, we leverage inductive conformal prediction and anomaly detection for developing a detection algorithm that has well-calibrated false alarm rate [11] and we compute the nonconformity scores in realtime using VAE and SVDD neural networks.

Conformal anomaly detection is used for sequential anomaly detection of time trajectories in [10]. A nonconformity measure based on nearest neighbors and Hausdorff distance provides good performance and robustness but it cannot be used for high-dimensional trajectories at runtime. Inductive techniques have been used for change-point detection in [5], [12] but do not consider high-dimensional inputs and realtime detection. A deep $k$-NN method based on conformal prediction that uses a nonconformity measure computed using the representation learned by each layer of the DNN classifier is presented in [24]. The method computes confidence metrics for evaluating the robustness of the DNN classifier and out-ofdistribution detection. Estimating the confidence and the uncertainty of neural network prediction is an important problem. In addition to conformal prediction, the problem is addressed using various techniques for computing a confidence or trust score [25], [26]. A promising research direction is to combine these with conformal prediction for improving the robustness and reliability of neural networks.

\section{CONCLUSIONS}

In this work, we demonstrated a method for out-ofdistribution detection in learning-enabled CPS. The method is based on inductive conformal prediction and anomaly detection but uses VAEs and SVDD to learn models to efficiently compute the nonconformity of new inputs relative to the training set and enable real-time detection of high-dimensional out-of-distribution inputs. Our evaluation is based on an AEBS and an SDEC implemented in an open source simulator for self-driving cars. The results demonstrate very small number of false positives and detection delay while the execution time is comparable to the execution time of the original LECs. Detection of out-of-distribution inputs does not necessarily imply large errors. An important question is whether it is possible to identify inputs that result in large errors. Evaluation with real-world data sets is also part of our future work.

\section{REFERENCES}

[1] C. E. Tuncali, G. Fainekos, H. Ito, and J. Kapinski, "Simulationbased adversarial test generation for autonomous vehicles with machine learning components," in 2018 IEEE Intelligent Vehicles Symposium.

[2] T. Dreossi, A. Donzé, and S. A. Seshia, "Compositional falsification of cyber-physical systems with machine learning components," in NASA Formal Methods Symposium. Springer, 2017, pp. 357-372.

[3] D. Hendrycks and K. Gimpel, "A baseline for detecting misclassified and out-of-distribution examples in neural networks," International Conference on Learning Representations, 2017.

[4] D. Hendrycks, M. Mazeika, and T. Dietterich, "Deep anomaly detection with outlier exposure," International Conference on Learning Representations, 2019.

[5] J. Smith, I. Nouretdinov, R. Craddock, C. Offer, and A. Gammerman, "Anomaly detection of trajectories with kernel density estimation by conformal prediction," in IFIP International Conference on Artificial Intelligence Applications and Innovations, 2014, pp. 271-280.

[6] C. Richter and N. Roy, "Safe visual navigation via deep learning and novelty detection," in Robotics: Science and Systems, 2017.

[7] R. McAllister, G. Kahn, J. Clune, and S. Levine, "Robustness to outof-distribution inputs via task-aware generative uncertainty," in International Conference on Robotics and Automation (ICRA), 2019.

[8] V. Vovk, A. Gammerman, and G. Shafer, Algorithmic learning in a random world. Springer Science \& Business Media, 2005.

[9] V. Balasubramanian, S.-S. Ho, and V. Vovk, Conformal prediction for reliable machine learning: theory, adaptations and applications. Newnes, 2014.

[10] R. Laxhammar and G. Falkman, "Online learning and sequential anomaly detection in trajectories," IEEE transactions on pattern analysis and machine intelligence, vol. 36, no. 6, pp. 1158-1173, 2013.

[11] _ " "Inductive conformal anomaly detection for sequential detection of anomalous sub-trajectories," Annals of Mathematics and Artificial Intelligence, vol. 74, no. 1-2, pp. 67-94, 2015.

[12] D. Volkhonskiy, E. Burnaev, I. Nouretdinov, A. Gammerman, and V. Vovk, "Inductive conformal martingales for change-point detection," Proceedings of Machine Learning Research, vol. 60, pp. 1-22, 2017.

[13] D. P. Kingma and M. Welling, "Auto-encoding variational bayes," in International Conference on Learning Representations, 2014.

[14] L. Ruff, R. Vandermeulen, N. Goernitz, L. Deecke, S. A. Siddiqui, A. Binder, E. Müller, and M. Kloft, "Deep one-class classification," in International Conference on Machine Learning, 2018, pp. 4393-4402.

[15] A. Dosovitskiy, G. Ros, F. Codevilla, A. Lopez, and V. Koltun, "CARLA: An open urban driving simulator," in Proceedings of the 1st Annual Conference on Robot Learning, 2017, pp. 1-16.

[16] A. Boloor, K. Garimella, X. He, C. Gill, Y. Vorobeychik, and X. Zhang, "Attacking vision-based perception in end-to-end autonomous driving models," arXiv preprint arXiv:1910.01907, 2019.

[17] V. Fedorova, A. Gammerman, I. Nouretdinov, and V. Vovk, "Plug-in martingales for testing exchangeability on-line," in 29th International Conference on Machine Learning, 2012, pp. 923-930.

[18] J. An and S. Cho, "Variational autoencoder based anomaly detection using reconstruction probability," Special Lecture on IE, (2)1, 2015.

[19] M. Basseville, I. V. Nikiforov et al., Detection of abrupt changes: theory and application. Prentice Hall Englewood Cliffs, 1993, vol. 104.

[20] T. P. Lillicrap, J. J. Hunt, A. Pritzel, N. Heess, T. Erez, Y. Tassa, D. Silver, and D. Wierstra, "Continuous control with deep reinforcement learning," in International Conference on Learning Representations, 2016.

[21] S. A. Seshia, D. Sadigh, and S. S. Sastry, "Towards verified artificial intelligence," arXiv preprint arXiv:1606.08514, 2016.

[22] X. Gu and A. Easwaran, "Towards safe machine learning for cps: infer uncertainty from training data," in 10th ACM/IEEE International Conference on Cyber-Physical Systems, 2019.

[23] S. Liang, Y. Li, and R. Srikant, "Enhancing the reliability of out-ofdistribution image detection in neural networks," in ICLR, 2017.

[24] N. Papernot and P. McDaniel, "Deep k-nearest neighbors: Towards confident, interpretable and robust deep learning," arXiv preprint arXiv:1803.04765, 2018.

[25] T. DeVries and G. W. Taylor, "Learning confidence for outof-distribution detection in neural networks," arXiv preprint arXiv:1802.04865, 2018

[26] H. Jiang, B. Kim, M. Guan, and M. Gupta, "To trust or not to trust a classifier," in Advances in neural information processing systems, 2018. 